\title{
Authors' Reply to Gandjour: "Can the EVIDEM Framework Tackle Issues Raised by Evaluating Treatments for Rare Diseases: Analysis of Issues and Policies, and Context-Specific Adaptation"
}

\author{
Monika Wagner $^{1} \cdot$ Mireille Goetghebeur ${ }^{1,2}$
}

Published online: 21 March 2017

(C) Springer International Publishing Switzerland 2017

We thank Dr. Gandjour [1] for his thoughtful comments and discussion of our work [2].

Over the last 10 years, we have been expounding, step by step, the many aspects of EVIDEM; initially, the userend, practical aspects (how to run it) and, more recently, in collaboration with ethicists, the conceptual aspects, i.e. the ethical underpinnings of its features and criteria. This work will soon be published in a book chapter [3], and commentaries and essays are also being prepared to further elucidate these fundamental aspects.

Dr. Gandjour's comment on convergence is particularly interesting as one premise of our approach is that there is a possibility of healthcare stakeholders converging under the ultimate goal of improving health, which can be defined according to three normative aspects: (i) identification of meaningful interventions for patients; (ii) consideration of population needs and equity; and (iii) healthcare system sustainability. A forth aspect is feasibility, i.e. the wisdom to make decisions adapted to the context. This was the foundation and motivation behind the design of EVIDEM.

As Dr. Gandjour noted, EVIDEM design does not presuppose an underlying ethical theory, but rather offers an interpretive frame for a decision situation and asks decision makers (be they patients, healthcare professionals, or

This is the reply to the article available at doi: 10.1007/s40273-015-0340-5.

Monika Wagner

monika.wagner@la-ser.com

1 LASER Analytica, 3100 Côte-Vertu Boulevard, Suite 230, St-Laurent, QC H4R 2J8, Canada

2 School of Public Health, University of Montreal, Montreal, QC, Canada policy makers) how they balance potentially conflicting ethical demands. While we believe these ethical norms per se are universally acknowledged, the way individuals adjudicate their relative importance (i.e. the personal value system) varies and, to a certain degree, can be expressed through weighting.

We agree that the definition of meaningful healthcare intervention has to cover the notion of autonomy. This notion is included in the framework but perhaps should be made more prominent and explicit. In addition to the decisional aspect of autonomy ('respecting the patient's voice'), the impact of a healthcare intervention on a patient's autonomy over a lifetime's trajectory needs to be further elaborated.

With respect to who decides, EVIDEM does not require, or advocate for, an expert approach (what was referred to as 'paternalistic'), but this is the current situation in most jurisdictions in which expert committees deliberate and make coverage decisions. Indeed, EVIDEM can be used in a consultative manner, as shown in the Colombian experience in which 211 individuals were consulted [4].

Weighting of EVIDEM's generic criteria is intended to help users reflect on what they value most, with awareness of each criterion's underlying ethical imperative. This is unlike the concept of preference elicitation, which is a feature of traditional multi-criteria decision analysis (MCDA). Weighting for preference elicitation also occurs in EVIDEM, but at the level of disease-specific subcriteria, such as intervention outcomes (e.g. number of episodes averted versus reduction of a disease marker).

Further theoretical development is certainly part of the EVIDEM endeavor. We are happy to move forward and contribute to the important debate on implicit value judgments and underlying motivations, and how reflection on 
the ethical underpinnings of decisions can be operationalized into pragmatic solutions that actually apply ethical reasoning to real life, which is what we have attempted to do collectively over the last 10 years. The mission of EVIDEM is to promote health by contributing a pragmatic approach to the necessary reflection on the legitimacy of decisions with respect to the goal that underlies the foundations of healthcare.

\section{Compliance with Ethical Standards}

Conflicts of interest Monika Wagner and Mireille Goetghebeur declare that they have no conflicts of interest directly relevant to the content of this letter.

Funding No funding was received for the preparation of this letter.

\section{References}

1. Gandjour A. Comment on: "Can the EVIDEM framework tackle issues raised by evaluating treatments for rare diseases: analysis of issues and policies, and context-specific adaptation". Pharmacoeconomics. 2017;34(3):285-301. doi:10.1007/s40273-015-0340-5.

2. Wagner M, Khoury H, Willet J, Rindress D, Goetghebeur M. Can the EVIDEM framework tackle issues raised by evaluating treatments for rare diseases: analysis of issues and policies, and context-specific adaptation. Pharmacoeconomics. 2016;34(3):285-301.

3. Goetghebeur M, Wagner M. Identifying values: a reflection on the ethical aspects of MCDA in healthcare decision making. In: Marsh K, Thokala P, Goetghebeur M, Baltussen B (eds) Healthcare decisions supported by multi-criteria decision analysis. Springer, Berlin; 2017.

4. Jaramillo HE, Goetghebeur M, Moreno-Mattar O. Testing multicriteria decision analysis for more transparent resource-allocation decision making in Colombia. Int $\mathbf{J}$ Technol Assess Health Care. 2016;32(4):307-314. 